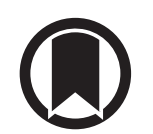

CrossMark

\title{
Triple therapy for all patients with severe symptomatic COPD at risk of exacerbations
}

\section{To the Editor:}

SUISSA and ARIEL [1] performed a post hoc analysis of TRIBUTE and IMPACT [2, 3], by "digitising" the figures to obtain values over each month of the cumulative incidence curves. They concluded that the lower rate of a first exacerbation with triple long-acting muscarinic antagonist/long-acting $\beta_{2}$-agonist/ inhaled corticosteroid (LAMA/LABA/ICS) therapy was exclusively due to a lower rate in the first month of treatment, while the rates were comparable to LAMA/LABA in the subsequent 11 months. They argue that this pattern of "depletion of susceptible individuals" might identify a small group of patients and suggest that only these patients could benefit from triple therapy, whereas the majority could be treated with LAMA/LABA without risk. They also found that the $42 \%$ lower mortality with triple therapy in IMPACT was localised in the first 4 months of follow-up, with no difference in the following 8 months. Based on these observations, they speculate that the subgroup who may benefit from triple therapy could be represented by patients with a history of asthma or eosinophilia, and they could be identified $a$ priori.

We acknowledge that in TRIBUTE there was a slightly higher incidence of first exacerbations shortly after initiation of treatment in the LAMA/LABA group. However, the analysis of SUISSA and ARIEL [1] was limited to the first event, excluding subsequent exacerbations. In a post hoc analysis of TRIBUTE based on multiple time-to-event methods (using the methodology developed by WEI et al. [4]), triple therapy not only prolonged the combined estimate of time to exacerbation versus LAMA/LABA (HR 0.84, 95\% CI 0.74-0.96; $\mathrm{p}=0.011$ ) but the effect size increased for multiple events, from a HR of 0.90 (95\% CI 0.76-1.06; $\mathrm{p}=0.219)$ for time to first exacerbation, to HR 0.80 (95\% CI $0.61-1.04 ; \mathrm{p}=0.096)$ for time to second and HR 0.58 (95\% CI $0.37-0.90 ; \mathrm{p}=0.014$ ) for time to third, with the effect on the third exacerbation reaching statistical significance [5]. We also observed a numerical reduction in all-cause mortality with triple therapy versus LAMA/LABA (2.7\% versus $2.1 \%)$ [2]. However, given the low rate of mortality, we do not believe it is appropriate to draw any conclusions about the distribution of such events in either study.

In response to the comments of SUISSA and ArIEL [1], we conducted additional post hoc analyses of the time to first moderate-or-severe chronic obstructive pulmonary disease (COPD) exacerbation in TRIBUTE. Only one patient had a history of asthma; it was therefore not possible to perform analyses stratified by this factor, but intrinsically this rules out the possibility that, at least in TRIBUTE, the observed early effect on exacerbations was due to patients with a history of asthma. When stratifying according to baseline blood eosinophil count (cut-off point 300 cells $\mu \mathrm{L}^{-1}$ ), a very similar pattern was observed in the first months in the two subgroups (figure $1 \mathrm{a}$ and $\mathrm{b}$ ).

Furthermore, our analysis by ICS use at study entry does not support the hypothesis that the superior efficacy shown by the triple combination is attributable to an ICS withdrawal effect (figure 1c and d). On the contrary, the subgroup that gained most benefit from triple therapy over the duration of the study was the group in which patients were not receiving ICS prior to study entry. Over the first 4 weeks, although there was an initial increase in exacerbation incidence in the LAMA/LABA group, this occurred in both the prior ICS and no prior ICS subgroups, with patients receiving LAMA/LABA approximately twice as likely to exacerbate than triple in both subgroups (probability of having experienced an exacerbation at 4 weeks with triple therapy versus LAMA/LABA: $4.4 \%$ versus $9.2 \%$ in the prior ICS subgroup, and $3.5 \%$ versus $7.6 \%$ in the no prior ICS subgroup).

@ERSpublications

In keeping with the latest international recommendations, we suggest using triple therapy in all patients with severe symptomatic COPD at increased risk of exacerbation who are not adequately treated by LAMA/LABA or LABA/ICS, independent of asthma history http://ow.ly/Bbsr30nXffO

Cite this article as: Papi A, Petruzzelli S, Vezzoli S, et al. Triple therapy for all patients with severe symptomatic COPD at risk of exacerbations. Eur Respir J 2019; 53: 1900147 [https://doi.org/10.1183/ 13993003.00147-2019]. 
a)

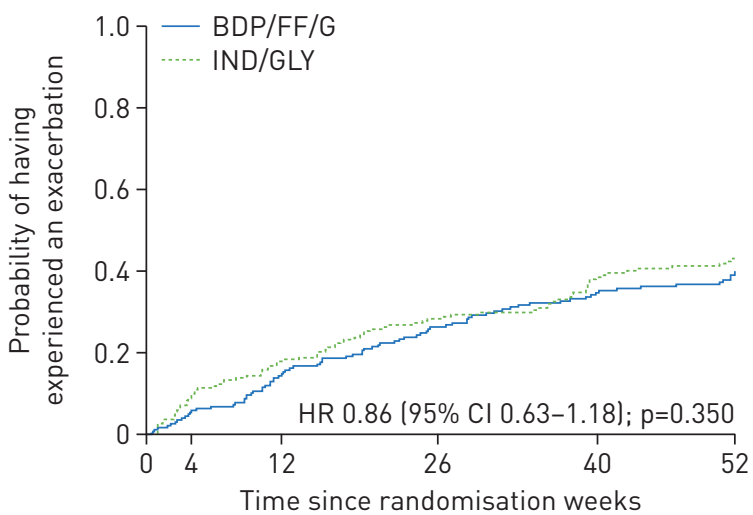

Patients at risk $n$

$\mathrm{BDP} / \mathrm{FF} / \mathrm{G} \quad 215200 \quad 180$

$\begin{array}{llll}\text { IND/GLY } & 208 & 185 & 166\end{array}$

$\begin{array}{lll}152 & 130 & 69 \\ 140 & 113 & 67\end{array}$

b)

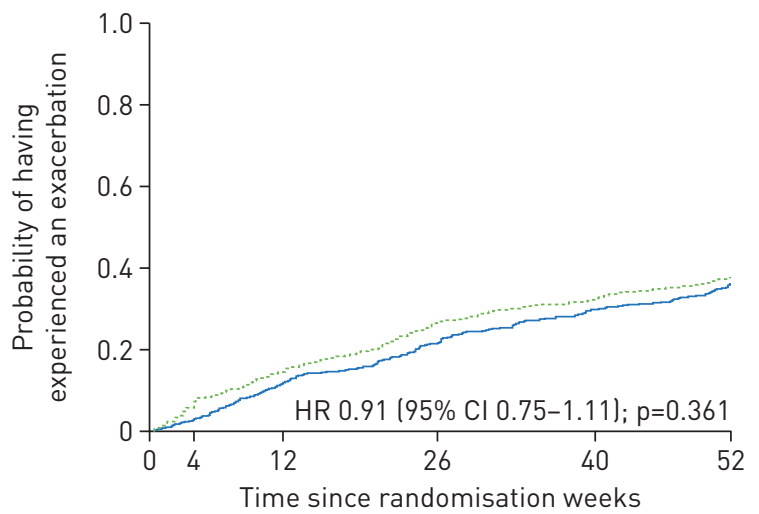

Patients at risk $n$

BDP/FF/G $\quad 547 \quad 524 \quad 469 \quad 403 \quad 354 \quad 206$

IND/GLY

383

343 d)

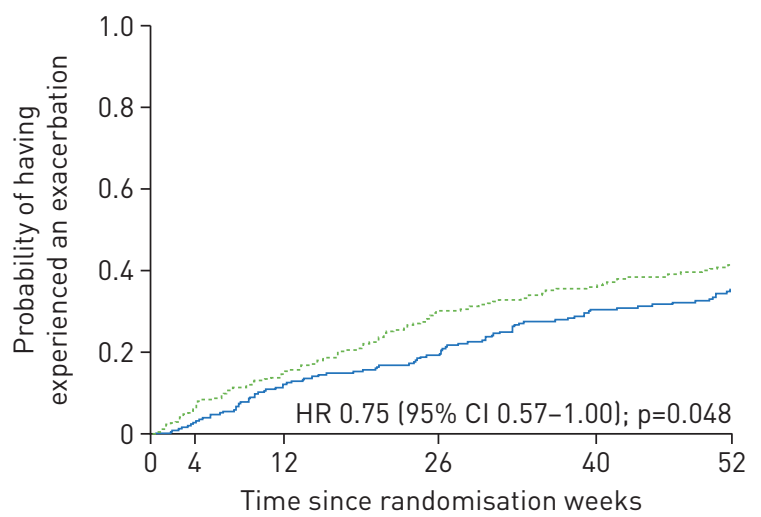

Patients at risk $n$

$\begin{array}{lllllll}\text { BDP/FF/G } & 260 & 252 & 225 & 197 & 166 & 91 \\ \text { IND/GLY } & 279 & 260 & 234 & 181 & 158 & 105\end{array}$

$279260 \quad 234$

18

105

Patients at risk $n$

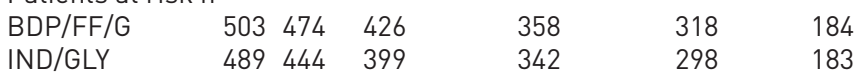

FIGURE 1 Kaplan-Meier curves of time to first moderate-or-severe chronic obstructive pulmonary disease (COPD) exacerbation in patients with baseline eosinophil counts al $\geqslant 300$ cells $\mu \mathrm{L}^{-1}$ and b) $<300$ cells. $\mu \mathrm{L}^{-1}$, and who prior to entering the study $\mathrm{c}$ ) were or d) were not receiving maintenance inhaled corticosteroids. Hazard ratios were estimated using a Cox proportional hazards model including the effects of treatment, country, number of COPD exacerbations in the previous year, severity of airflow limitation and smoking status. BDP/FF/G: beclometasone dipropionate/formoterol fumarate/glycopyrronium; IND/GLY: indacaterol/glycopyrronium.

Thus, the speculation of SUISSA and ARIEL [1] about the TRIBUTE results is not supported by evidence. In addition to preventing exacerbations, in TRIBUTE and IMPACT triple therapy had a significant benefit on health-related quality of life and, more importantly, showed a signal toward improved survival compared to bronchodilator therapy without ICS $[2,3,6]$. There is also a risk that by following the suggestions of SUISSA and ARIEL [1], ICS withdrawal might be considered in patients who do not exacerbate during the first month of treatment with triple therapy. However, we do not know whether withdrawing ICS could trigger subsequent exacerbations at a later time. Even expanding the observation period from 1 month to 12 months may not be adequate, given that data from SPIROMICS indicate patients can have events 2 or 3 years after the index date [7]. Overall, randomised controlled trials have shown that the benefits of reducing the risk of exacerbations with these two triple therapies compared to LAMA/LABA outweigh the increase in risk of adverse effects including pneumonia $[2,3,8]$. Thus, in keeping with the latest international Global Initiative for Chronic Obstructive Lung Disease recommendations [9], we suggest using triple therapy in patients with severe, symptomatic COPD at increased risk of exacerbation who are not adequately treated by LAMA/LABA or LABA/ICS, with the exception being patients at increased risk of side-effects with a specific component, i.e. LAMA, LABA or ICS $[8,10]$.

Alberto Papi $\oplus^{1}$, Stefano Petruzzelli ${ }^{2}$, Stefano Vezzoli ${ }^{2}$, George Georges ${ }^{3}$ and Leonardo M. Fabbri $\oplus^{1,4}$

${ }^{1}$ Section of Cardiorespiratory and Internal Medicine, Dept of Medical Sciences, University of Ferrara, Ferrara, Italy. ${ }^{2}$ Global Clinical Development, Chiesi Farmaceutici SpA, Parma, Italy. ${ }^{3}$ Global Clinical Development, Chiesi USA, Inc., Cary, NC, USA. ${ }^{4}$ COPD Center, Institute of Medicine, Sahlgrenska University Hospital, University of Gothenburg, Gothenburg, Sweden. 
Correspondence: Alberto Papi, Respiratory Medicine, University of Ferrara, Via Rampari di San Rocco, 27, Ferrara, 44121, Italy. E-mail: ppa@unife.it

Received: Jan 212019 | Accepted after revision: Feb 032019

Acknowledgements: Writing support was provided by David Young (Young Medical Communications and Consulting Ltd, Horsham, UK). This support was funded by Chiesi Farmaceutici SpA.

Conflict of interest: A. Papi reports grants, personal fees, non-financial support and other from Chiesi, AstraZeneca, GlaxoSmithKline, Boehringer Ingelheim, Menarini, Novartis, Zambon, Mundipharma and TEVA, and grants from Sanofi, outside the submitted work. S. Petruzzelli is employed by Chiesi Farmaceutici SpA, the sponsor of the studies. S. Vezzoli is employed by Chiesi Farmaceutici SpA, the sponsor of the studies. G. Georges reports other support from Chiesi USA, Inc., during the conduct of the study; and other support from Chiesi USA, Inc., outside the submitted work. L.M. Fabbri reports personal fees and non-financial support from AstraZeneca, GSK, Novartis, Menarini, Boehringer Ingelheim, Zambon and Pearl Therapeutics, non-financial support from Dompe, and grants, personal fees and non-financial support from Chiesi, outside the submitted work.

Support statement: This study was funded by Chiesi Farmaceutici. Funding information for this article has been deposited with the Crossref Funder Registry.

\section{References}

$1 \quad$ Suissa S, Ariel A. Triple therapy trials in COPD: a precision medicine opportunity. Eur Respir J 2018; 52: 1801848.

2 Papi A, Vestbo J, Fabbri L, et al. Extrafine inhaled triple therapy versus dual bronchodilator therapy in chronic obstructive pulmonary disease (TRIBUTE): a double-blind, parallel group, randomised controlled trial. Lancet 2018; 391: 1076-1084

3 Lipson DA, Barnhart F, Brealey N, et al. Once-daily single-inhaler triple versus dual therapy in patients with COPD. N Engl J Med 2018; 378: 1671-1680.

4 Wei LJ, Lin DY, Weissfeld L. Regression analysis of multivariate incomplete failure time data by modeling marginal distributions. J Am Stat Assoc 1989; 84: 1065-1073.

5 Fabbri L, Singh D, Vestbo J, et al. Extrafine beclometasone dipropionate, formoterol fumarate, glycopyrronium (BDP/FF/G) and exacerbation recurrence: post-hoc analysis of the TRIBUTE study. Eur Respir J 2018; 52: Suppl 62, PA4381.

6 Vestbo J, Fabbri LM, Papi A, et al. Inhaled corticosteroid containing combinations and mortality in COPD. Eur Respir J 2018; 52: 1801230.

7 Han MK, Quibrera PM, Carretta EE, et al. Frequency of exacerbations in patients with chronic obstructive pulmonary disease: an analysis of the SPIROMICS cohort. Lancet Respir Med 2017; 5: 619-626.

8 Agusti A, Fabbri LM, Singh D, et al. Inhaled corticosteroids in COPD: Friend or foe? Eur Respir J 2018; 52: 1801219.

9 Global Initiative for Chronic Obstructive Lung Disease. Global strategy for the diagnosis, management, and prevention of Chronic Obstructive pulmonary disease. 2019. https://goldcopd.org/wp-content/uploads/2018/11/ GOLD-2019-v1.7-FINAL-14Nov2018-WMS.pdf

10 Vanfleteren LEGW, Anders Ullman A, Nordenson A, et al. Triple therapy (ICS/LABA/LAMA) in COPD: thinking out of the box. ERJ Open Res 2019; 5: 00185-2018.

\section{Triple therapy in COPD: only for the right patient}

From the author:

We thank A. Papi and co-workers for their letter on our examination of the recent single inhaler triple therapy trials. Their additional analyses provide further evidence for our interpretation that these trials

@ERSpublications

The TRIBUTE and IMPACT trials comparing triple therapy with LAMA/LABA in COPD have methodological limitations that inherently favor triple therapy; use with caution by targeting the right drug for the right patient in terms of both benefit and risk http://ow.ly/Bbsr30nXffO

Cite this article as: Suissa S, Ariel A. Triple therapy in COPD: only for the right patient. Eur Respir J 2019; 53: 1900394 [https://doi.org/10.1183/13993003.00394-2019]. 


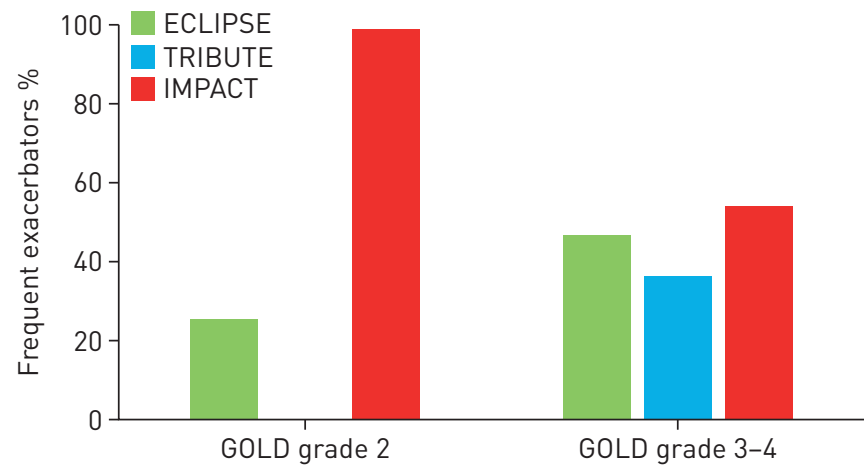

FIGURE 1 Proportion of frequent exacerbators ("D" patients) by Global Initiative for Chronic Obstructive Lung Disease (GOLD) grade, for the ECLIPSE cohort, and the TRIBUTE and IMPACT trials. Since ECLIPSE reported data separately on patients with any two or more exacerbations during the year and those with severe exacerbations, we approximated the number of " $D$ " patients as the sum of those with two or more exacerbations and $50 \%$ of those with severe exacerbations.

provide significant opportunities for a precision medicine approach to chronic obstructive pulmonary disease (COPD) treatment [1]

To start with, it is important to point out three distinctions between the TRIBUTE and IMPACT trial designs, as our editorial comments applied equally to both $[2,3]$. First, the TRIBUTE trial included only patients with severe-to-very severe airflow limitation (Global Initiative for Chronic Obstructive Lung Disease (GOLD) grade 3-4; forced expiratory volume in $1 \mathrm{~s}\left(\mathrm{FEV}_{1}\right)<50 \%$ ) and at least one moderate-to-severe exacerbation in the previous year, resulting in a study population with an average FEV 1 predicted of $36 \%$ and $9 \%$ with bronchodilator reversibility. $37 \%$ were "frequent exacerbators" (GOLD "D" patients; personal communication A. Papi), namely those with $\geqslant 2$ exacerbations or one severe exacerbation in the previous year. In contrast, the IMPACT trial also included patients with moderate airflow limitation (GOLD grade 2; FEV1 50-80\%) provided they were frequent exacerbators, resulting in an unusual "asthma-like" and "ICS-susceptible" study population with an average FEV1 of 45\% predicted, $18 \%$ with bronchodilator reversibility and 70\% frequent exacerbators [3].

These exacerbation patterns are quite different from the ECLIPSE general COPD cohort [4]. Indeed, for GOLD grade 3-4, the TRIBUTE and IMPACT study populations are close to expected (figure 1). However, for GOLD grade 2, the IMPACT study population greatly over represents the frequent exacerbators.

Secondly, both trials allowed patients using pre-study ICS, who accounted for $\sim 65 \%$ and $70 \%$ of patients included in TRIBUTE and IMPACT, respectively. However, TRIBUTE excluded patients already on triple therapy while IMPACT allowed such patients who made up $\sim 40 \%$ of the study population.

Thirdly, both trials imposed that all patients discontinue their maintenance therapy. In TRIBUTE, this discontinuation was followed by a 2 -week run-in period during which all patients received the long-acting muscarinic antagonist/long-acting $\beta_{2}$-agonist (LAMA/LABA) comparator, after which they were randomised to LAMA/LABA/inhaled corticosteroid (ICS) or LAMA/LABA. This could have depleted patients who benefit from ICS and favoured the selection of patients more likely to benefit from LAMA/ LABA [5]. In contrast, patients in IMPACT continued their pre-study maintenance treatment during a 2-week run-in period, after which they abruptly discontinued this therapy and were randomised to LAMA/LABA/ICS or LAMA/LABA (or LABA/ICS).

The fact that the "depletion of susceptibles" paradigm led to a greater surge in the rate of a first exacerbation in the first month after randomisation to LAMA/LABA in IMPACT (15.0 per 100 per month) than in TRIBUTE (7.1 per 100 per month) may be surprising in view of the more severe population selected in TRIBUTE [1]. We propose, however, that the main reason for this difference lies in the important distinctions in study designs, particularly the inclusion of more frequent exacerbators and the more abrupt discontinuation of pre-study ICS in IMPACT.

The post hoc analysis of the TRIBUTE data, addressing not only the first exacerbation but also the subsequent ones, suggests that the reduction in exacerbations with triple therapy is sustained for the second and third exacerbations. However, our argument specifically addressed the early first exacerbations (in the first month), not all first exacerbations as analysed by A. Papi and co-workers. Nevertheless, we believe that this post hoc analysis is consistent with the early surge in exacerbation in the LAMA/LABA 
group, with a majority withdrawn from ICS, which probably identifies the frequent exacerbators who would benefit from triple therapy $[4,6,7]$.

The post hoc stratification of the TRIBUTE data, depicting the Kaplan-Meier curves by pre-study ICS use, actually confirms our assertion. The subgroup of patients who used pre-study ICS clearly shows the first-month surge in exacerbation among the LAMA/LABA group, with no difference in the incidence over the remaining 11 months. There is no such early surge for those who were not on pre-study ICS, and the incidence remains lower with triple therapy than with LAMA/LABA throughout the entire follow-up. We submit that this stratified analysis provides a proof of concept in identifying the proper intervention group for investigating the add-on effect of triple therapy, namely patients who were already on LAMA or LABA or both, but not on ICS. The larger IMPACT study, with data properly stratified by patient inclusion groups (GOLD 2 frequent exacerbators versus GOLD 3-4), as well as by pre-study ICS use and baseline eosinophil level, may further clarify the role of triple therapy in COPD.

In all, the complex methodological issues involved in pharmacotherapy trials in COPD, particularly those involving ICS, require careful study design and interpretation [8]. As illustrated by the TRIBUTE and IMPACT trials, this includes a proper choice of study population, avoiding "asthma-like" patients and interventions affected by drug withdrawal. Alternatively, the trials could include pre-specified subgroup analyses by these factors to address these issues. Such considerations will provide more evidence for a precision medicine approach to COPD treatment, namely better targeting of the right treatment for the right patient in terms of both benefit and risk [9].

Samy Suissa ${ }^{1}$ and Amnon Ariel ${ }^{2}$

${ }^{1}$ Center for Clinical Epidemiology, Lady Davis Institute - Jewish General Hospital, and the Deps of Epidemiology and Biostatistics and of Medicine, McGill University, Montreal, QC, Canada. ${ }^{2}$ Lung Unit, Emek Medical Center, Clalit Healthcare Services, Afula, Israel.

Correspondence: Samy Suissa, Centre for Clinical Epidemiology, Jewish General Hospital, 3755 Cote Ste-Catherine, Montreal, QC, Canada H3T 1E2. E-mail: samy.suissa@mcgill.ca

Received: Feb 252019 | Accepted: Feb 262019

Acknowledgements: We thank A. Papi and L.M. Fabbri for providing the data on frequent exacerbators in the TRIBUTE trial.

Conflict of interest: S. Suissa reports personal fees from AstraZeneca, and grants from Boehringer-Ingelheim Novartis, outside the submitted work. A. Ariel reports personal fees from AstraZeneca, GlaxoSmithKline and Kamada, and personal fees and non-financial support from Boehringer Ingelheim, outside the submitted work.

\section{References}

1 Suissa S, Ariel A. Triple therapy trials in COPD: a precision medicine opportunity. Eur Respir J 2018; 52: 1801848.

2 Papi A, Vestbo J, Fabbri L, et al. Extrafine inhaled triple therapy versus dual bronchodilator therapy in chronic obstructive pulmonary disease (TRIBUTE): a double-blind, parallel group, randomised controlled trial. Lancet 2018; 391: 1076-1084.

3 Lipson DA, Barnhart F, Brealey N, et al. Once-daily single-inhaler triple versus dual therapy in patients with COPD. N Engl J Med 2018; 378: 1671-1680.

4 Hurst JR, Vestbo J, Anzueto A, et al. Susceptibility to exacerbation in chronic obstructive pulmonary disease. N Engl J Med 2010; 363: 1128-1138.

5 Suissa S. Run-in bias in randomised trials: the case of COPD medications. Eur Respir J 2017; 49: 1700361.

6 Hurst JR, Donaldson GC, Quint JK, et al. Temporal clustering of exacerbations in chronic obstructive pulmonary disease. Am J Respir Crit Care Med 2009; 179: 369-374.

7 Suissa S, Dell'Aniello S, Ernst P. Long-term natural history of chronic obstructive pulmonary disease: severe exacerbations and mortality. Thorax 2012; 67: 957-963.

8 Suissa S, Ernst P, Vandemheen KL, et al. Methodological issues in therapeutic trials of COPD. Eur Respir J 2008; 31: 927-933.

9 Suissa S, Ernst P. Precision medicine urgency: the case of inhaled corticosteroids in COPD. Chest 2017; 152: 227-231. 